\title{
Effect of Probiotics on Pharmacokinetics of Orally Administered Acetaminophen in Mice
}

\author{
Jeon-Kyung Kim, Min Sun Choi, Jin-Ju Jeong, Su-Min Lim, In Sook Kim, Hye Hyun Yoo, \\ and Dong-Hyun Kim
}

Departments of Life and Nanopharmaceutical Sciences and Pharmacy, Kyung Hee University, Dongdaemun-gu, Seoul, Republic of Korea (J.-K.K., J.-J.J., S.-M.L., D.-H.K.); and Institute of Pharmaceutical Science and Technology and College of Pharmacy, Hanyang University, Ansan, Gyeonggi-do, Republic of Korea (M.S.C., I.S.K., H.H.Y.)

Received June 27, 2017; accepted November 29, 2017

\begin{abstract}
Orally administered probiotics change gut microbiota composition and enzyme activities. Thus, coadministration of probiotics with drugs may lead to changes in the pharmacokinetic parameters of the drugs. In this study, we investigated the pharmacokinetics of acetaminophen in mice treated with probiotics. Oral administration of probiotics changed the gut microbiota composition in the mice. Of these probiotics, Lactobacillus reuteri $\mathrm{K} 8$ increased the numbers of clostridia, bifidobacteria, and enterococci, and Lactobacillus rhamnosus $\mathrm{K} 9$ decreased the number of bifidobacteria, determined by culturing in selective media. Next, we performed a pharmacokinetic study of acetaminophen in mice orally treated with K8 and K9 for 3 days. Treatment with $\mathrm{K} 8$ reduced the area under the curve (AUC) of orally administered acetaminophen to $68.4 \%$ compared with normal
\end{abstract}

\section{Introduction}

Orally administered drugs are absorbed through the gastrointestinal (GI) tract into the blood. Absorption depends on the solubility, stability, and permeability of the drug as well as on its metabolism by enzymes secreted by the body and gut microbiota (Davis, 2005; Lennernäs and Abrahamsson, 2005; Al-Hilal et al., 2013; Kim, 2015). Nevertheless, the effects of gut microbiota on the absorption of drugs from the GI into the blood have not been studied sufficiently. The ability of gut bacteria to metabolize xenobiotics, particularly drugs, is comparable to that of any organs in the body, including the liver (Sousa et al., 2008; Saad et al., 2012; Kim, 2015).

Drug metabolism has been thought mainly to occur in the liver. However, recent studies (Joh and Kim, 2010; Tralau et al., 2015) have reported that orally administered xenobiotics are metabolized by gut microbiota before their absorption into the blood. The metabolic reactions performed by the gut microbiota are quite different from those of the liver; the liver primarily catalyzes the formation of hydrophilic metabolites through oxidative and conjugative reactions, including

This research was supported by a grant from the Ministry of Food and Drug Safety of Korea [16182MFDS416].

J.-K.K. and M.S.C. contributed equally to this work.

https://doi.org/10.1124/dmd.117.077222. control mice, whereas $\mathrm{K} 9$ did not affect the AUC of acetaminophen. Oral administration to mice of K8, which degraded acetaminophen, increased the degradation of acetaminophen by gut microbiota, whereas $\mathrm{K} 9$ treatment did not affect it. Treatment with $\mathrm{K} 8$ increased the number of $L$. reuteri adhered in the upper small intestine, whereas the number of $L$. rhamnosus was not affected by treatment with K8 or K9. K8 increased the number of cyanobacteria, whereas K9 increased the number of deferribacteres. These results suggest that the intake of probiotics may make the absorption of orally administered drugs fluctuate through the disturbance of gut microbiota-mediated drug metabolism and that the subsequent impact on microbiota metabolism could result in altered systemic concentrations of the intact drug.

ABBREVIATIONS: ABQ, $N$-acetyl-p-benzoquinoneimine; AUC, area under the curve; CA, Clostridium agar; CFU, colony-forming units; EA, mEnterococcus agar; GI, gastrointestinal; HPLC, high-performance liquid chromatography; LC/MS/MS, liquid chromatography-tandem mass spectroscopy; LC/QTOF/MS, liquid chromatography quadrupole time-of-flight mass spectrometer; qPCR, quantitative polymerase chain reaction; $\mathrm{SPE}$, solid-phase extraction; $t_{1 / 2}$, half-life. 
Eubacterium rectale A-44 or Enterobacter amnigenus AR (Kim et al., 1992; Kwon et al., 1999). The pharmacokinetic parameters of orally administered acetaminophen may be affected by endogenous and exogenous factors of fluctuating gut microbiota.

Probiotics, frequently found in yogurt, kimchi, and human gut microbiota, are considered beneficial microorganisms because they exhibit beneficial biologic effects for constipation, diarrhea, colitis, and diabetes and suppress the harmful enzyme production of gut microbiota. Therefore, the use of probiotics is increasing in medical practice (Goldin and Gorbach, 2008; Wasilewski et al., 2015; Bron et al., 2017). Probiotics can in fact simultaneously be used in treatment with therapeutic drugs. But, they interact with many drugs in the gut and may cause the pharmacokinetic parameters of orally administered drugs to fluctuate (Lee et al., 2012a; Stojančević et al., 2014); some probiotics affect drug metabolism involving certain drug-metabolizing enzymes, for example, the metabolism of nifedipine by CYP3A in the intestinal mucosa or the metabolism of sulfasalazine by gut microbiota (Kato et al., 2007). Therefore, to understand the effects of probiotics on the pharmacokinetics of frequently used drugs, we evaluated the effect of probiotics on the pharmacokinetics of orally administered acetaminophen in mice.

\section{Materials and Methods}

Materials. Acetaminophen, formic acid, and dextrose were purchased from Sigma-Aldrich (St. Louis, MO). QIAamp Fast DNA Stool Mini Kit was purchased from Qiagen (Hilden, Germany). BL agar was purchased from Nissui Pharmaceutical Co. (Tokyo, Japan). m-Enterococcus agar (EA) was purchased from BD (Franklin Lakes, NJ). Clostridium agar (CA) was purchased from MB Cell (Los Angeles, CA). High-performance liquid chromatography (HPLC) grade acetonitrile was purchased from J.T. Baker (Phillipsburg, NJ). Distilled water was prepared using a Milli-Q purification system (Millipore, Billerica, MA). Lactobacilli (K1, Lactobacillus acidophilus KCTC3140; K2, Lactobacillus casei KCTC3109; K3, Lactobacillus gasseri KCTC3148; K4; Lactobacillus delbueckii subsp. bulgaricus KCTC3635; K5, Lactobacillus helveticus KCTC3545; K6, Lactobacillus fermentum KCTC3112; K7, Lactobacillus paracasei KCTC13169; K8, Lactobacillus reuteri KCTC3679; K9, Lactobacillus rhamnosus KCTC3237; and K10, Lactobacillus salivarius KCTC5922) were purchased from Korea Collection for Type Cultures (Daejeon, Korea).

Animals. Male C57BL/6 mice (weight, 22-25 g; age, 8 weeks) were purchased from RaonBio Inc. (Seoul, Korea) housed in a wire cage under controlled conditions (light/dark, every 12 hours; temperature, $20-22^{\circ} \mathrm{C}$; humidity, $50 \% \pm 10 \%$ ), and acclimated for 1 week before the experiments. Mice were fed standard laboratory chow and water ad libitum. The experiment was performed according to the protocol of the Kyung Hee University Guideline for Laboratory Animal Care and Usage. The experiment protocol was ethically approved by the Committee for the Care and Use of Laboratory Animals [KHPASP(SE)-16-027]. Pharmacokinetic experiments were performed in accordance with the Hanyang University Guidelines for Laboratory Animal Care and Use and approved by the Committee for the Care and Use of Laboratory Animals in the College of Pharmacy, Hanyang University (2016-0151).

Analysis of Gut Microbiota Composition. Mice were orally gavaged probiotics $\left[1 \times 10^{9}\right.$ colony-forming units $\left.(\mathrm{CFU}) / \mathrm{mouse}\right]$ once a day for 5 days. The fresh feces (approximately $0.1 \mathrm{~g}$ ) were collected 6 hours before probiotics treatment, 1 or 3 days after the final gavage of probiotics. The analysis of gut microbiota composition was performed using selective media according to the method of Lim et al. The fresh feces were gently suspended in $0.9 \mathrm{ml}$ of cold GAM (Gifu Anaerobic) broth (BD, Sparks, MD). The suspended solution was diluted with the broth and inoculated into CA for clostridia, BL for bifidobacteria, and EA for enterococci. BL agars were anaerobically cultured for 2 and 3 days, respectively. EA was aerobically cultured for 24 hours.

For the analysis of gut microbiota composition by quantitative polymerase chain reaction (qPCR), fecal DNA was extracted from the fresh feces using QIAampFast DNA Stool Mini Kit (Qiagen) and analyzed Proteobacteria, Actinobacteria, Bacteroidetes, and Firmicutes by qPCR. qPCR was performed according to the method of Yang et al. (2015), utilizing a Qiagen thermal cycler, which used SYBR premixed agents, as per the instructions from Takara Bio Inc. (Kusatsu, Japan), as follows: activation of DNA polymerase at $95^{\circ} \mathrm{C}$ for 30 seconds and 40 cycles of amplification at $95^{\circ} \mathrm{C}$ for 5 seconds and at $63^{\circ} \mathrm{C}$ for 30 seconds. The normalized expression of the assayed genes, with respect to bacterial recombinant RNA, was computed for all samples using the Microsoft (Redmond, WA) Excel data spreadsheet. Primers were used as follows: Firmicutes: forward, 5'-GGAGYATGTGGTTTAATTCGAAGCA-3', reverse, 5'-AGCTGACGACAACCATGCAC-3'; Bacteroidetes: forward, 5'-GTTTAATTCGATGATACGCGAG-3', reverse, 5' -TTAASCCGACACCTCACGG-3'; Actinobacteria: forward, 5'-TGTAGCGGTGGAATGCGC-3', reverse, 5' -AATTAAGCCACATGCTCCGCT-3'; $\gamma / \delta$-Proteobacteria: forward, 5'-GCTAACGCATTAAGTRYCCCG-3', reverse, 5'-GCCATGCRGCACCTGTCT-3'; and bacterial recombinant RNA: forward, 5'-AGAGTTTGATCCTGGCTCAG-3', reverse, 5'-AAGGAGGTGWTCCARCC-3'.

Preparation of Mouse Fecalase and Intestinal Enzyme Fraction. The feces of each mouse dosed with or vehicle after the treatment with probiotics (approximately $0.3 \mathrm{~g}$ ) were collected in plastic tubes, suspended in $2.7 \mathrm{ml}$ of cold saline, and centrifuged at $500 \mathrm{~g}$ and $4^{\circ} \mathrm{C}$ for 5 minutes. The supernatant was sonicated for 2 minutes five times and then centrifuged at $10,000 \mathrm{~g} 4^{\circ} \mathrm{C}$ for 20 minutes. The supernatant was used for the enzyme activity assay. Small intestines and colons were removed from the mice, washed with phosphatebuffered saline three times, homogenized in $50 \mathrm{mM}$ Tris- $\mathrm{HCl}$ buffer ( $\mathrm{pH} 7.4$ ), and
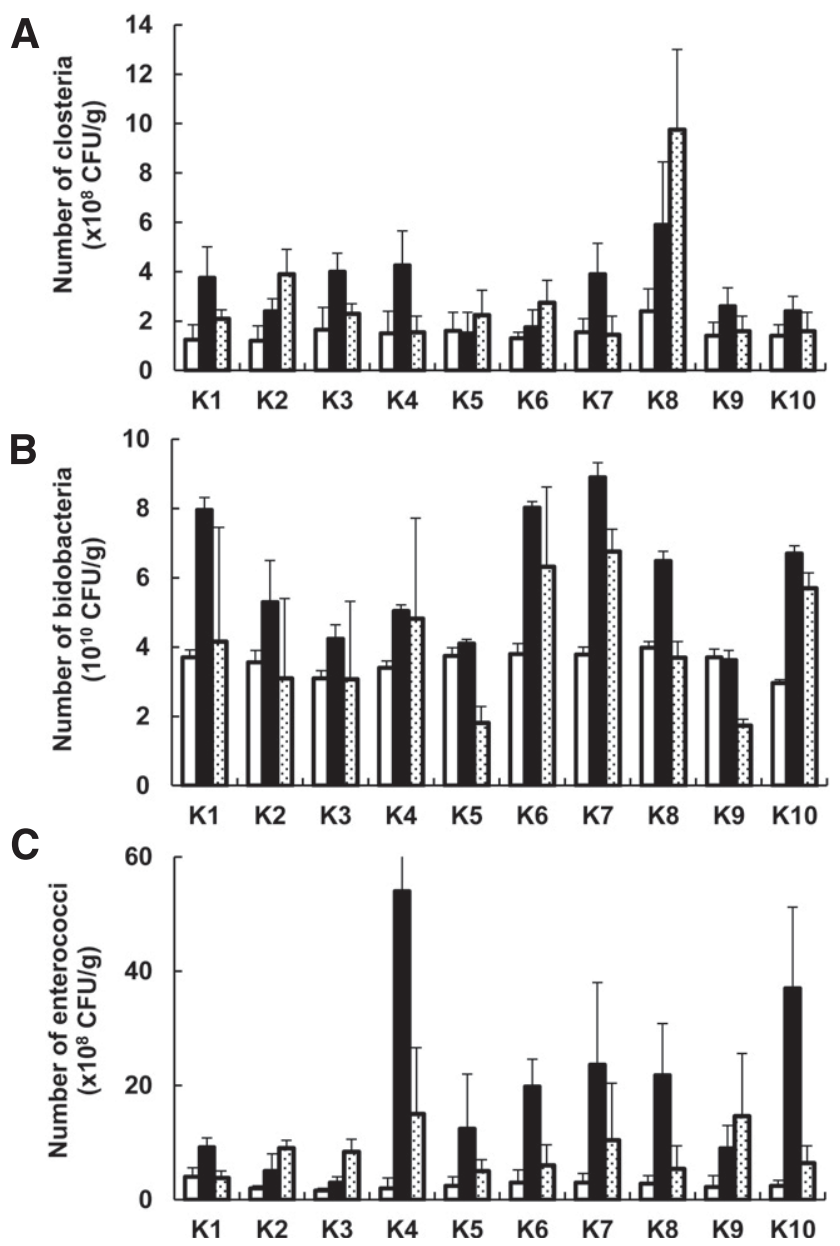

Fig. 1. Effects of commercially available probiotics on the composition of gut microbiota in mice. (A) Effects on the number of bifidobacteria grown in BL agar plates. (B) Effects on the number of clostridia grown in CA plates. (C) Effects on the number of enterococci grown in EA agar plates. Probiotics $\left(1 \times 10^{9} \mathrm{CFU} / \mathrm{mouse}\right)$ was orally administered to mice once a day for 3 days. The fresh stools were collected before, and 1 and 3 days after the final treatment with probiotics. The plates were anaerobically incubated at $37^{\circ} \mathrm{C}$ for 2 days. $\square$, before; 1 day; 3 days. 
centrifuged at $10,000 \mathrm{~g}$ and $4^{\circ} \mathrm{C}$ for 30 minutes. The resulting supernatants were used as a crude enzyme fraction of intestinal tissues.

Acetaminophen-Metabolizing Activity Assay. The reaction mixture (total volume of $2.5 \mathrm{ml}$ ), which consisted of $0.25 \mathrm{ml}$ of $0.25 \mathrm{mM}$ acetaminophen, $2 \mathrm{ml}$ of $0.1 \mathrm{M}$ potassium phosphate buffer $(\mathrm{pH} 7.0)$. and $0.25 \mathrm{ml}$ of fecal suspension or a probiotic K8 or K9 cultured in MRS broth $\left(1 \times 10^{11} \mathrm{CFU} / \mathrm{ml}\right)$, was incubated at $37^{\circ} \mathrm{C}$ for 12 hours, stopped by the addition of $1.5 \mathrm{ml}$ of $\mathrm{MeOH}$, and centrifuged (3000 $\mathrm{g}$ for 15 minutes). The amount of acetaminophen in the resulting supernatant was assayed by HPLC or thin-layer chromatography.

Enzyme Activity Assay in the Feces and Intestinal Tissues. For the activity assay of fecal $\beta$-glucuronidase, and sulfatase, the reaction mixture (total volume of $0.5 \mathrm{ml}$ ), which contained $0.1 \mathrm{ml}$ of $1 \mathrm{mmol} / \mathrm{l} p$-nitrophenyl- $\beta$-D-glucuronide for $\beta$-glucuronidase or $p$-nitrophenyl sulfate for sulfatase, $0.3 \mathrm{ml}$ of $0.05 \mathrm{~mol} / 1$ phosphate buffer, $\mathrm{pH} 7.0$, and $0.1 \mathrm{ml}$ of the fecalase or intestinal enzyme fraction, was incubated at $37^{\circ} \mathrm{C}$ for 20 minutes. For the activity assay of fecal arylsulfate sulfotransferase, the reaction mixture (total volume of $0.5 \mathrm{ml}$ ), which contained $0.1 \mathrm{ml}$ of $1 \mathrm{mmol} / 1 p$-nitrophenyl sulfate, $0.1 \mathrm{ml}$ of $1 \mathrm{mmol} / 1$ acetaminophen, $0.2 \mathrm{ml}$ of $0.05 \mathrm{~mol} / \mathrm{l}$ phosphate buffer, $\mathrm{pH} 7.0$, and $0.1 \mathrm{ml}$ of the fecal suspension or enzyme solution, was incubated at $37^{\circ} \mathrm{C}$ for 30 minutes. The reaction was stopped by the addition of $0.5 \mathrm{ml}$ of $10 \mathrm{mmol} / \mathrm{l} \mathrm{NaOH}$ and centrifuged at $3000 \mathrm{~g}$ for 2 minutes; the absorbance was measured at $405 \mathrm{~nm}$ (UV-visible spectrophotometer, catalog \#UV-1201; Shimadzu, Columbia, MD). Enzyme activity was indicated as the amount required to catalyze the formation of $1.0 \mathrm{nmol} p$ nitrophenol/h under standard assay conditions. Specific activity was defined as millimoles per hour per gram of wet feces.

Fecal Sample Analysis by LC/QTOF/MS. The fecal incubation samples were analyzed using LC/QTOF/MS. The instrument consisted of an Agilent 1260 HPLC system and an Agilent 6530 QTOF mass spectrometer with an electrospray interface (Agilent Technologies, Santa Clara, CA). Chromatographic separation was performed on a Poroshell 120 EC-C18 column $(2.7 \mu \mathrm{m}, 2.1 \times$ $100 \mathrm{~mm}$; Agilent Technologies). Column oven temperature was maintained at $40^{\circ} \mathrm{C}$. The HPLC mobile phases consisted of $0.1 \%$ formic acid in distilled water (A) and $90 \%$ acetonitrile in $0.1 \%$ formic acid (B). A gradient program was used at a flow rate of $0.2 \mathrm{ml} / \mathrm{min}$, as follows: $5 \% \mathrm{~B}$ to $85 \%$ B for 15 minutes, followed by a 7-minute re-equilibration. Mass detection was performed in the positive ion mode. The drying gas (nitrogen) temperature was set at $350^{\circ} \mathrm{C}$. The drying gas flow was set at $10 \mathrm{l} / \mathrm{min}$. The nebulizer pressure was set at $20 \mathrm{psi}$. The capillary and fragmentor voltages were 3500 and $110 \mathrm{~V}$, respectively. High-purity nitrogen was introduced into the collision cell as fragmentation gas.

Pharmacokinetic Experiments. Mice pretreated with probiotics or vehicle $(n=6)$ were administered an i.v. $(0.5 \mathrm{mg} / \mathrm{kg})$ or an oral $(10 \mathrm{mg} / \mathrm{kg})$ dose of acetaminophen dissolved in saline $(5 \mathrm{mg} / \mathrm{ml}) 24$ hours after completing the 5 consecutive days of treatment with $1 \%$ dextrose (control) or each probiotic (K8 or K9) dissolved in $1 \%$ dextrose $\left(1 \times 10^{9} \mathrm{CFU} /\right.$ mouse $)$. The whole-blood samples were taken from the orbital vein and put into heparinized tubes at $0.03,0.16,0.5$, $1,2,4,6$, and 8 hours after i.v. injection or at $0.16,0.5,1,2,4,6$, and 8 hours after oral administration. The plasma was harvested by centrifugation at $13,200 \mathrm{rpm}$ for 5 minutes and stored at $-70^{\circ} \mathrm{C}$ until analyzed for acetaminophen.

Blood Sample Preparation and Calibration Curves. A 20- $\mu 1$ aliquot of plasma was deproteinized with $20 \mu \mathrm{l}$ of acetonitrile containing $10 \mathrm{ng} / \mathrm{ml}$ bupropion (internal standard) to which $20 \mu \mathrm{l}$ of distilled water was added. The sample was vigorously vortex mixed, and then centrifuged at 13,200 rpm for 5 minutes. The resulting supernatant was transferred to LC vials, and a 5- $\mu$ l aliquot was injected into the LC/MS/MS analysis system. The calibration curve of acetaminophen in mouse plasma was constructed using seven calibration standards at concentrations of 5, 10, 20, 100, 500, 1000, and $6000 \mathrm{ng} / \mathrm{ml}$. Calibration curves were generated by plotting the peak area ratios of the analyte to the internal standard versus the analyte concentrations, and the calibration curve equation was generated by linear least-squares regression. The regression coefficient $\left(r^{2}\right)$ was greater than 0.999. The accuracy and the precision of the calibration standard curves were reliable (less than $\pm 15 \%$ relative S.D.) for all the concentration points tested.
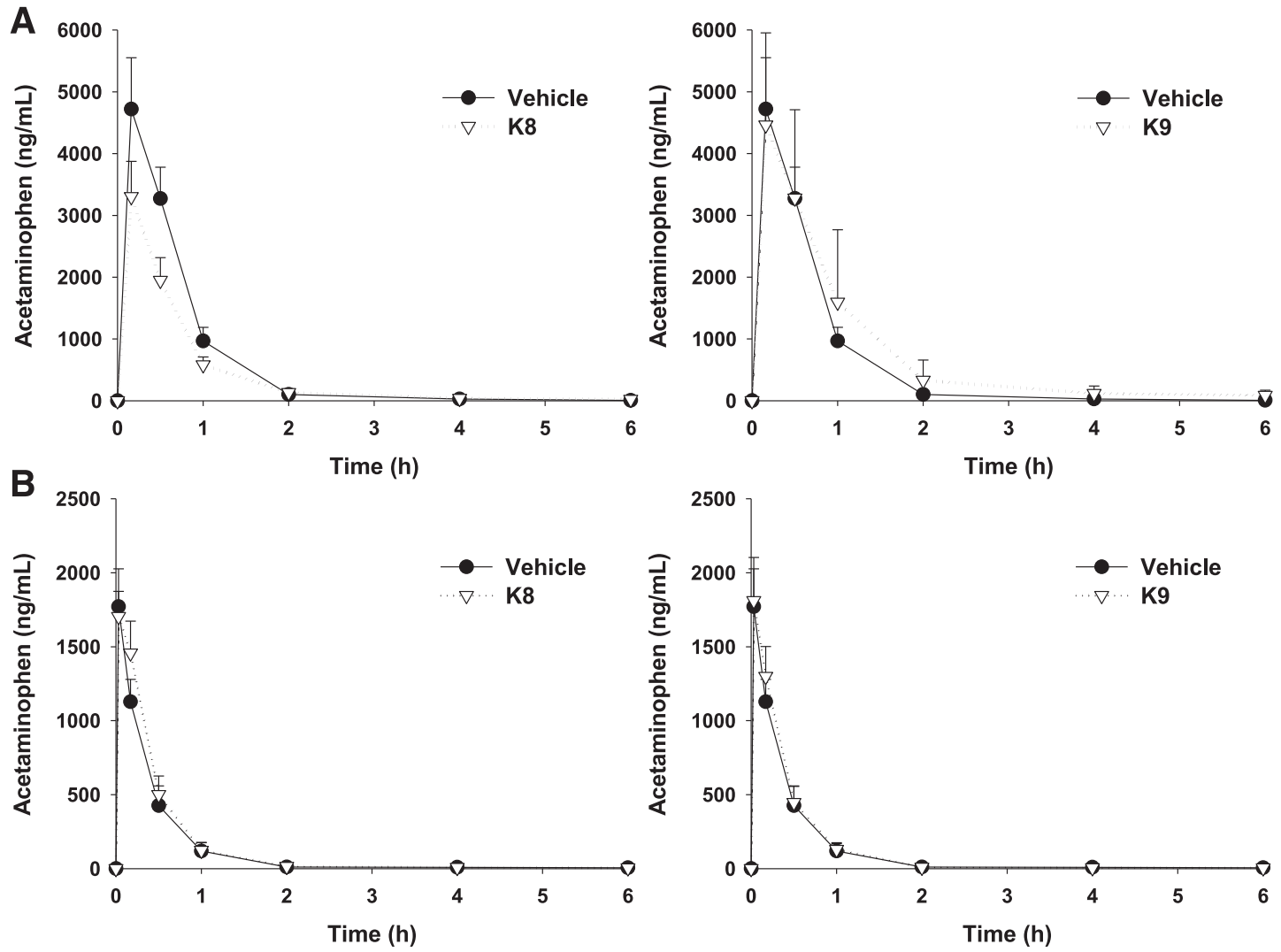

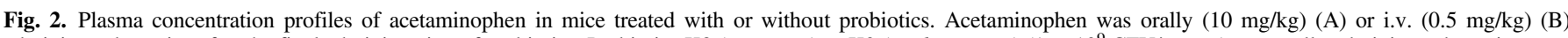

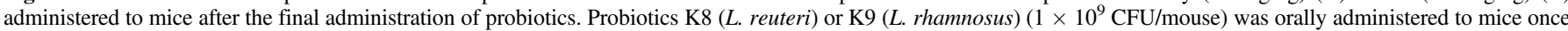
a day for 3 days before treatment with acetaminophen. Data represent the mean \pm S.D. $(n=6)$. 
TABLE 1

Pharmacokinetic parameters of acetaminophen after oral administration in mice pretreated with or without probiotics $(n=6)$

\begin{tabular}{lccc}
\hline \multirow{2}{*}{ Parameters } & \multicolumn{3}{c}{ Acetaminophen $(10 \mathrm{mg} / \mathrm{kg})$} \\
\cline { 2 - 4 } & Control & K8 & K9 \\
\hline$T_{\max }(\mathrm{h})$ & $0.16 \pm 0.00$ & $0.16 \pm 0.00$ & $0.16 \pm 0.00$ \\
$C_{\max }(\mu \mathrm{g} / \mathrm{ml})$ & $4.72 \pm 0.83$ & $3.30 \pm 0.57^{*}$ & $3.96 \pm 0.96$ \\
$\mathrm{AUC}(\mu \mathrm{g} / \mathrm{h}$ per milliliter $)$ & $3.51 \pm 0.47$ & $2.40 \pm 0.26^{* *}$ & $3.65 \pm 0.98$ \\
$\mathrm{Cl} / \mathrm{F}(1 / \mathrm{h}$ per kilogram $)$ & $2.89 \pm 0.39$ & $4.14 \pm 0.49^{* *}$ & $2.89 \pm 0.71$ \\
$t_{1 / 2}(\mathrm{~h})$ & $1.09 \pm 0.35$ & $1.65 \pm 0.70$ & $1.22 \pm 0.24$ \\
\hline
\end{tabular}

$T_{\max }$, time taken to reach maximum. Data are presented as the mean \pm S.D.

$* P<0.05$ vs. control; $* * P<0.01$ vs. control.

LC/MS/MS Analysis for Pharmacokinetic Samples. The LC/MS/MS system consisted of an Agilent 1260 Infinity HPLC system with an Agilent 6460 triple-quadrupole mass spectrometer equipped with an electrospray ionization source (Agilent Technologies). Chromatographic separation was achieved with a Halo C8 column $(2.7 \mu \mathrm{m}, 2.1 \times 100 \mathrm{~mm}$; VWR, Radnor, PA); oven temperature was maintained at $40^{\circ} \mathrm{C}$. The mobile phase consisted of $0.1 \%$ formic acid in distilled water (A) and $0.1 \%$ formic acid in acetonitrile (B). A gradient program was used as follows: $10 \%$ B to $90 \%$ B at 2 minutes, to $10 \%$ B at 0.1 minute, and held at $10 \%$ B for 4.9 minutes, with a flow rate of $0.3 \mathrm{ml} / \mathrm{min}$. The drying gas (nitrogen) temperature was set at $300^{\circ} \mathrm{C}$, the drying gas flow at $100 \mathrm{l} / \mathrm{min}$, the nebulizer pressure at $20 \mathrm{psi}$, and the capillary voltage at $3500 \mathrm{~V}$. Fragment voltages were set at $90 \mathrm{~V}$ for acetaminophen and $130 \mathrm{~V}$ for bupropion. Multiple-reaction monitoring detection was used, with nitrogen as the collision gas; the precursor-product ion pairs monitored were $152>110$ for acetaminophen and $240>184$ for bupropion.

Pharmacokinetic Analysis. The $C_{\mathrm{max}}$, the time taken to reach maximum, and the area under the plasma concentration-time curve from time 0 to last values for acetaminophen were estimated directly from the plasma concentration-time profiles. The Phoenix WinNonlin Enterprise Program version 5.3 (Pharsight Inc., St. Louis, MO) was used with a noncompartmental statistical model to determine other kinetic parameters of the mouse plasma samples.

Acetaminophen Metabolite Profiling in Plasma, Urine, and Feces. After an overnight fast, the mice were orally dosed with $10 \mathrm{mg} / \mathrm{kg}$ acetaminophen and housed individually in metabolic cages equipped with a urine and feces separator. Heparinized samples of blood were collected at 30 minutes after dose administration. Urine and feces were collected and weighed at the following intervals: before dose administration and $0-24$ hours after dose administration. All samples were stored separately at $-20^{\circ} \mathrm{C}$ until analysis. In the plasma samples, $40 \mu \mathrm{l}$ of acetonitrile was added to $40 \mu \mathrm{l}$ of plasma. Then it was vortex mixed for 30 seconds and centrifuged of 13,200 rpm for 5 minutes, and a 50- $\mu$ l aliquot of supernatant was diluted to $50 \mu 1$ of distilled water. After urine sample collections, the urine samples were purified with solid-phase extraction (SPE) using an Oasis HLB 96-well $\mu$ Elution Extraction Plate (Waters, Milford, MA). Urine was loaded onto an SPE plate that was pretreated with $1 \mathrm{ml}$ methanol. SPE columns were washed twice with water and eluted in $1 \mathrm{ml}$ of methanol. The elution was collected and evaporated to dryness under nitrogen stream at $45^{\circ} \mathrm{C}$. These residues were dissolved in $100 \mu \mathrm{l}$ of $20 \%$ methanol in water. For feces samples, three volumes of methanol were added to the feces, followed by homogenization and then

TABLE 2

Pharmacokinetic parameters of acetaminophen after i.v. injection in mice pretreated with or without probiotics $(n=6)$

\begin{tabular}{lccc}
\hline \multirow{2}{*}{ Parameters } & \multicolumn{3}{c}{ Acetaminophen $(0.5 \mathrm{mg} / \mathrm{kg})$} \\
\cline { 2 - 4 } & Control & K8 & K9 \\
\hline AUC $(\mu$ g/h per milliliter $)$ & $0.76 \pm 0.13$ & $0.85 \pm 0.16$ & $0.80 \pm 0.11$ \\
Cl $(1 / \mathrm{h}$ per kilogram) & $0.67 \pm 0.14$ & $0.60 \pm 0.10$ & $0.63 \pm 0.08$ \\
$t_{1 / 2}(\mathrm{~h})$ & $0.94 \pm 0.15$ & $1.00 \pm 0.16$ & $0.94 \pm 0.08$ \\
Vz $(1 / \mathrm{kg})$ & $0.92 \pm 0.26$ & $0.86 \pm 0.17$ & $0.86 \pm 0.15$ \\
MRT $(\mathrm{h})$ & $0.58 \pm 0.05$ & $0.53 \pm 0.04$ & $0.53 \pm 0.05$ \\
\hline
\end{tabular}

Data are presented as the mean \pm S.D. centrifugation at $1000 \mathrm{~g}$ for 5 minutes. The supernatant was filtered using a $0.2-\mu \mathrm{m}$ polytetrafluoroethylene syringe filter and diluted with water. The samples were analyzed using $\mathrm{LC} / \mathrm{MC} / \mathrm{MC}$ analyses according to a previously reported method (An et al., 2012).

Statistics. All the data were expressed as the mean \pm S.D., and statistical significance was analyzed by Student's $t$ test or analysis of variance coupled with Tukey's post hoc test, with statistical significance set at $P<0.05$.

\section{Results}

Effects of Probiotics on the Pharmacokinetics of Acetaminophen. To understand the effects of probiotics on the pharmacokinetics of acetaminophen in mice, we first selected probiotics that influenced the gut microbiota composition. Ten kinds of probiotics were gavaged into mice, the fresh feces were cultured using selective media, and the consequent changes in the number of the representative gut bacteria were periodically analyzed (Fig. 1). Most of the probiotics increased the number of bifidobacteria grown in BL, whereas $\mathrm{K} 5$ and $\mathrm{K} 9$ gradually reduced the number of bifidobacteria grown in BL. K8 significantly increased the number of clostridia grown in CA. Several probiotics including $\mathrm{K} 4$ and $\mathrm{K} 10$ significantly increased the number of enterococci grown in EA. Taken collectively, K8, and $\mathrm{K} 9$ were supposed to be the most characteristic probiotics, and thus, we selected these two probiotics for subsequent studies, where we investigated the effects of K8 and K9 on the pharmacokinetics of acetaminophen (Fig. 2).
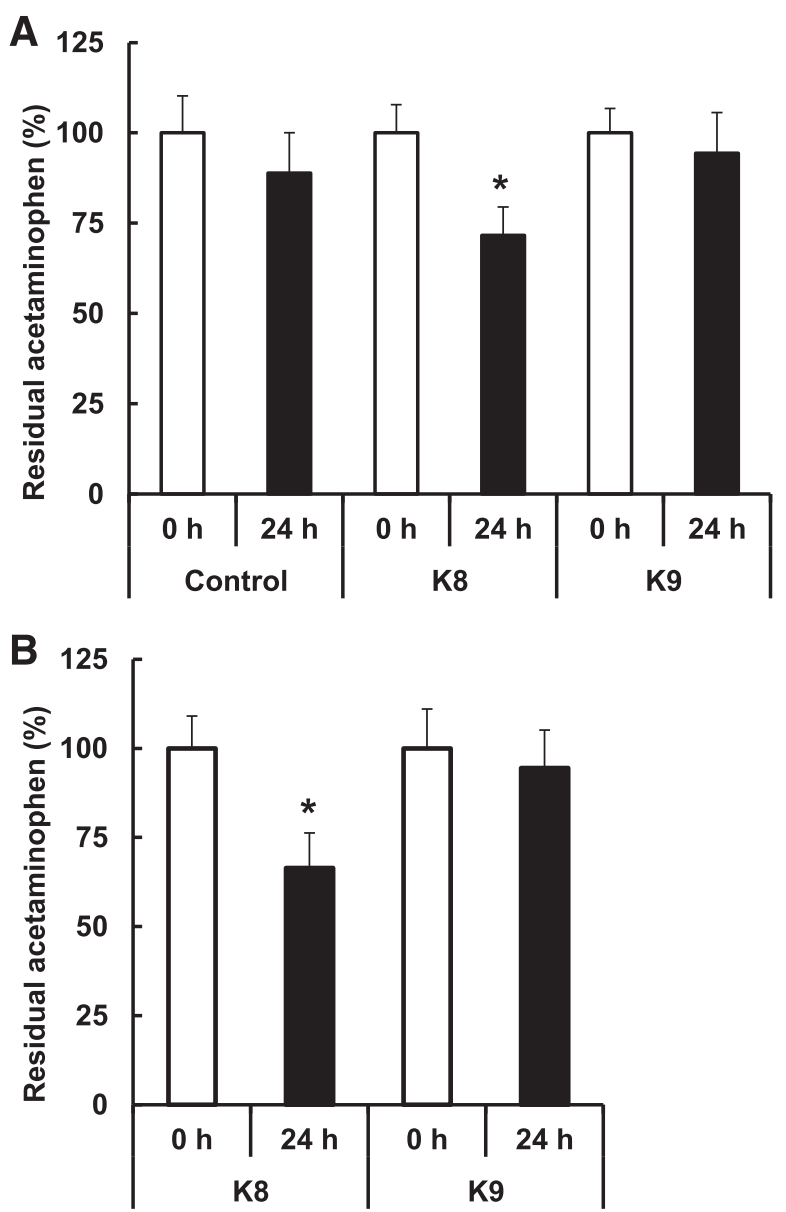

Fig. 3. Effects of probiotics on the acetaminophen-metabolizing activity of gut microbiota. (A) Fecal acetaminophen-metabolizing activity in mice after treatment of probiotics. (B) The acetaminophen-metabolizing activity of K8 and K9. Data are shown as the mean \pm S.D. $(n=6)$. $* P<0.05$ vs. control group. 
TABLE 3

The concentration of acetaminophen in the contents from different parts of the GI tract of control and probiotics-treated mice after oral administration of acetaminophen $(n=5)$

The GI contents were collected at $1 \mathrm{~h}$ after the administration of acetaminophen.

\begin{tabular}{lcccc}
\hline & \multicolumn{4}{c}{ Acetaminophen $(\mathrm{ng} / \mathrm{ml})$} \\
\cline { 2 - 5 } & Stomach & Small Intestine & Cecum & Large Intestine \\
\hline Control & $3902.6 \pm 3648.2$ & $159.5 \pm 96.7$ & $2.1 \pm 2.2$ & $0.0 \pm 0.0$ \\
K8 & $574.3 \pm 979.6$ & $156.0 \pm 166.6$ & $447.1 \pm 485.8$ & $2.2 \pm 2.6$ \\
K9 & $8147.0 \pm 12,794.2$ & $304.7 \pm 278.5$ & $173.1 \pm 133.9$ & $14.2 \pm 20.4$ \\
\hline
\end{tabular}

Data are presented as the mean \pm S.D.

The plasma concentration levels of acetaminophen were determined after oral administration to the vehicle- or probiotic-treated mice. The mean plasma concentration-time profiles of acetaminophen in the control and probiotic-treated mice groups are shown in Fig. 2A, and the resultant pharmacokinetic parameters are described in Table 1. The $C_{\max }$ and area under the curve (AUC) values of acetaminophen in the control group were $4.72 \pm 0.83 \mu \mathrm{g} / \mathrm{ml}$ and $3.51 \pm 0.47 \mu \mathrm{g} / \mathrm{h}$ per milliliter, respectively. As for K8-treated mice, the $C_{\max }$ and AUC values were $3.30 \pm 0.57 \mu \mathrm{g} / \mathrm{ml}$ and $2.40 \pm 0.26 \mu \mathrm{g} / \mathrm{h}$ per milliliter, respectively; both parameters significantly decreased compared with those in the control group. Meanwhile, the $C_{\max }$ and AUC values in the K9-treated mice did not show any significant difference from the control values, although some differences were observed between their mean values. As for $\mathrm{Cl} / \mathrm{F}$ and half-life $\left(t_{1 / 2}\right)$, the $\mathrm{K} 8$-treated group showed the increased values of $\mathrm{Cl} / \mathrm{F}$ compared with other groups. However, the $t_{1 / 2}$ values were comparable among the three groups.

Meanwhile, when acetaminophen was administered i.v. after probiotics treatment, the plasma concentration profile of acetaminophen was not changed (Fig. 2B). These data indicate that the pharmacokinetic changes of acetaminophen in probiotics-treated mice resulted mainly from the alterations in the GI tract. The resulting pharmacokinetic parameters are summarized in Table 2 .

Effects of Probiotics on the Acetaminophen Metabolism in the Gut. To understand how probiotic K8 could inhibit the absorption of acetaminophen from the GI tract into the blood, we first incubated
A

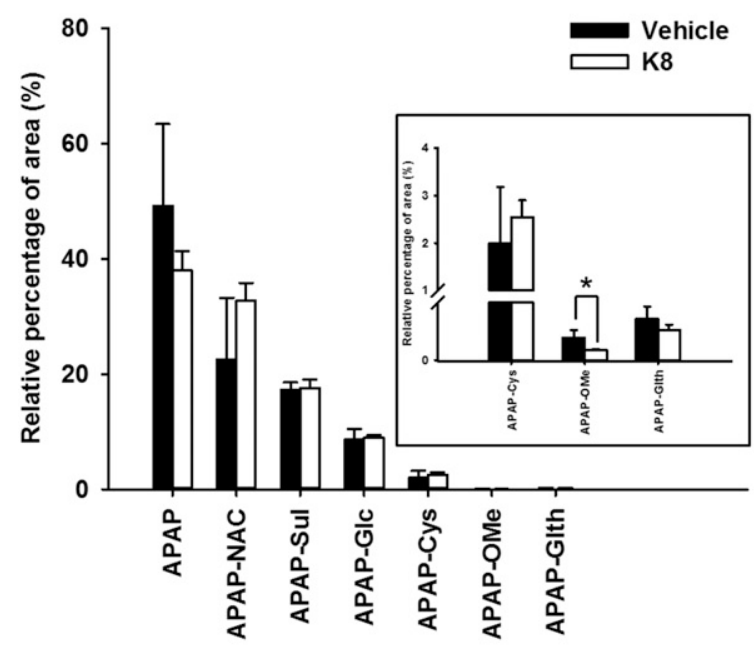

B Urine

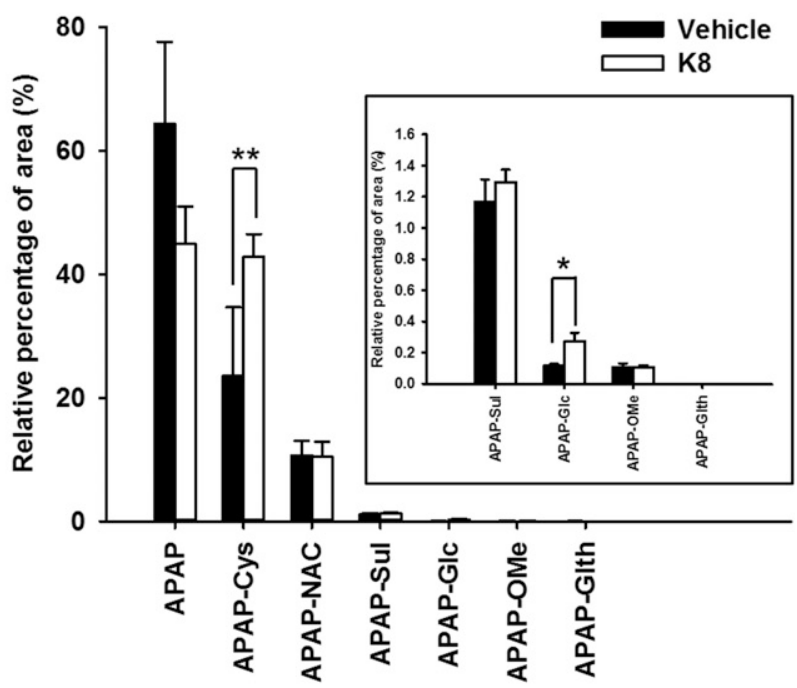

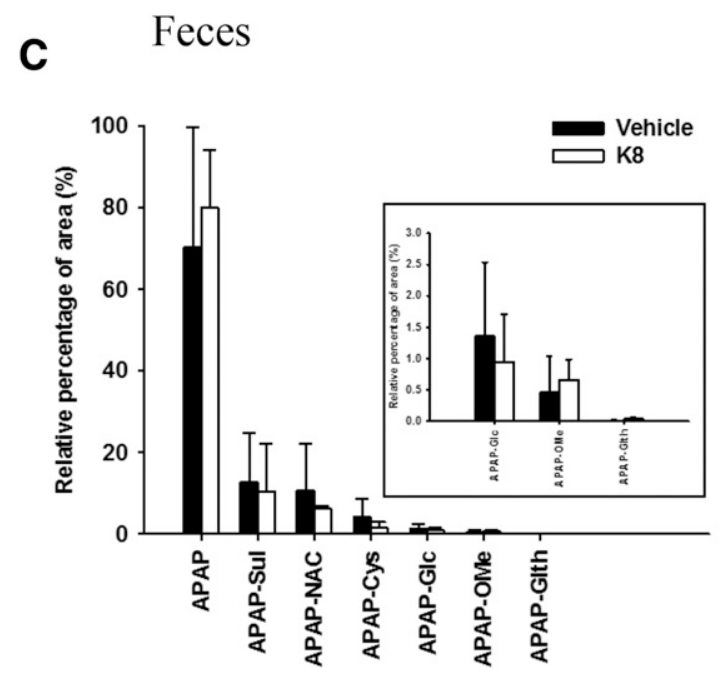

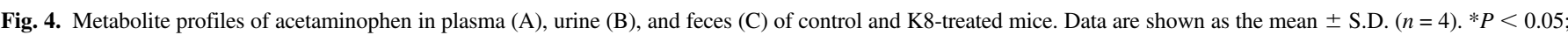

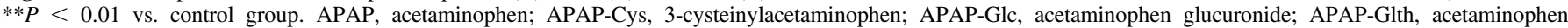
glutathione; APAP-NAC, 3-(N-acetyl- $L$-cystein- $S$-yl) acetaminophen; APAP-OME, 3-methoxy acetaminophen; APAP-Sul, acetaminophen sulfate. 

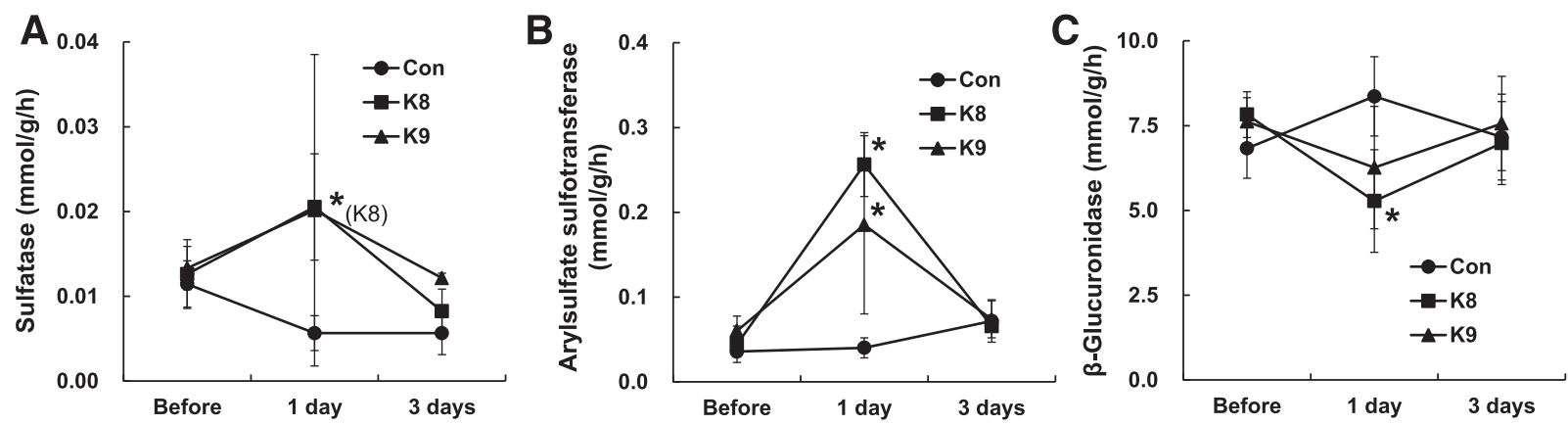

Fig. 5. Effects of probiotics on the fecal sulfatase (A), arylsulfate sulfotransferase (B), and $\beta$-glucuronidase (C) activities in mice treated with or without probiotics. Probiotics $\left(1 \times 10^{9} \mathrm{CFU} / \mathrm{mouse}\right)$ was orally administered to mice once a day for 3 days. The fresh stools were collected before, and 1 and 3 days after the final treatment with probiotics. Data are shown as the mean \pm S.D. $(n=6) . * P<0.05$ vs. control group.

acetaminophen with mouse fecal suspension for 24 hours and the amount of acetaminophen remaining was measured (Fig. 3A). The remaining amount of acetaminophen decreased after the 24-hour incubation; mouse fecal suspension significantly degraded acetaminophen. Based on these data, the acetaminophen-metabolizing activities of mouse fecal suspension were measured as $0.029 \pm 0.028 \mathrm{nmol} / \mathrm{h}$ per milligram.

Next, to investigate the in vivo effects of probiotics on the metabolic activity of acetaminophen by gut microbiota, we orally administered probiotics to mice and measured fecal acetaminophen-metabolizing activity 24 hours after the final oral administration of the probiotics (Fig. 3A). The oral administration of probiotics caused some of the activity to fluctuate; treatment with $\mathrm{K} 8$ significantly increased the acetaminophen-metabolizing activity compared with normal control mice, whereas K9 treatment did not significantly influence the activity. We also measured the acetaminophen-metabolizing activity of probiotics themselves. Although the acetaminophen-degrading activity of the probiotics was weaker than that of the fecal suspension, they also degraded acetaminophen (Fig. 3B). Of the probiotics tested, K8 significantly increased the metabolic activity of acetaminophen. These findings on the changes in gut microbial acetaminophen-metabolizing activities by probiotics are consistent with the pharmacokinetic results after probiotic treatment.

In addition, to confirm the effects of $\mathrm{K} 8$ on the gut microbial metabolism of acetaminophen, we measured the acetaminophen concentration in the contents of different parts of the GI tract after oral administration of acetaminophen to control or probiotics-treated mice (Table 3). The mean acetaminophen concentration in the stomach of K8-treated mice was lower than that of control and K9-treated mice, but statistical significance was not shown. There was no difference in the concentrations in the small intestine between $\mathrm{K} 8$ and control groups. Meanwhile, the mean acetaminophen concentration in the cecum was somewhat higher than that in the other two groups, which suggests that K8 treatment might stimulate the peristaltic movements of the GI tract and the acetaminophen moved more quickly to the lower part of the intestine.

Metabolite Profiles of Acetaminophen in Plasma, Urine, and Feces. The concentrations of acetaminophen metabolites were measured in plasma, urine, and feces of control and K8-treated mice. Acetaminophen and its six metabolites were determined, and their levels were plotted as a relative percentage based on peak area (Fig. 4). In plasma and urine, the percentage of acetaminophen in K8-treated mice was lower than that in control mice, which is consistent with the plasma pharmacokinetic results. The portion of urinary concentration of the cysteine conjugate was elevated about 2-fold in K8-treated mice. In addition, there were significant differences in the ratios of the urinary concentration of acetaminophen glucuronide and plasma concentration of the $O$-methylated metabolite. In feces, the conjugate metabolite ratios were generally decreased by $\mathrm{K} 8$ treatment, which reveals that $\mathrm{K} 8$ or K8inducing alteration of gut microbiota might promote the degradation of the conjugate metabolites by gut microbial enzymes.

Effects of Probiotics on the Enzymes Involved in the Deconjugation of Acetaminophen. As for the enzymes that may be involved in the conjugation of acetaminophen, both $\mathrm{K} 8$ and $\mathrm{K} 9$ significantly increased fecal sulfatase and arylsulfate sulfotransferase activities.
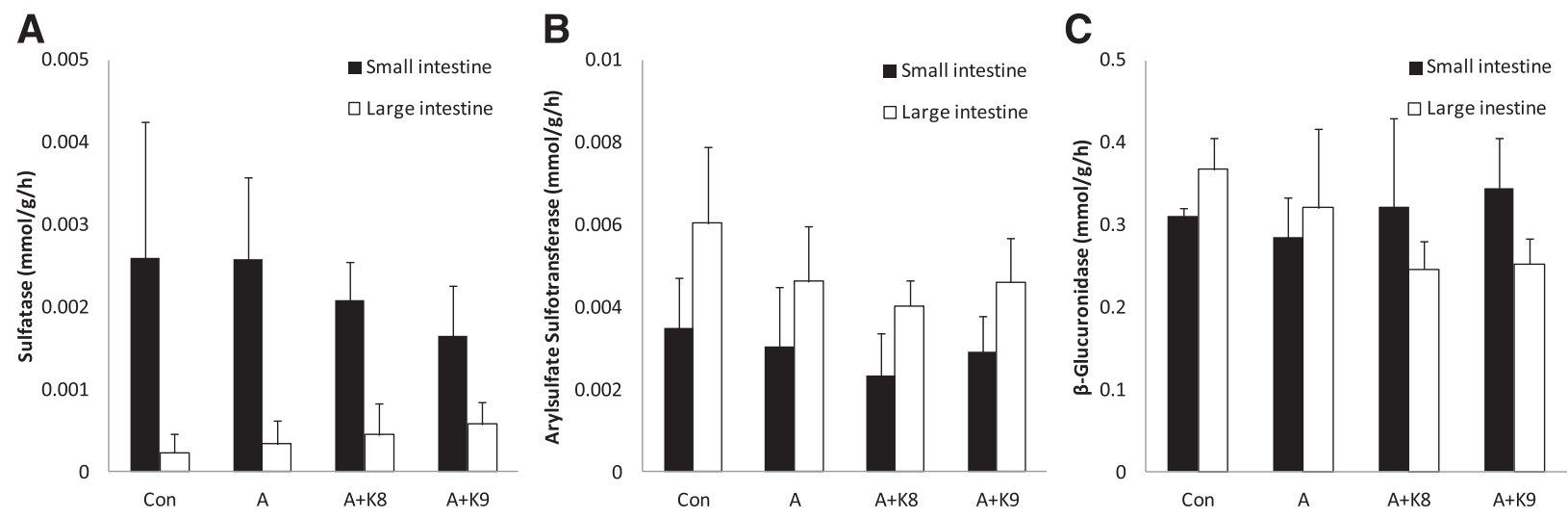

Fig. 6. Effects of probiotics on the intestinal sulfatase (A), arylsulfate sulfotransferase (B), and $\beta$-glucuronidase (C) activities in mice. A, acetaminophen was orally administered; $\mathrm{A}+\mathrm{K} 8$, acetaminophen was orally administered to mice treated with $\mathrm{K} 8$; $\mathrm{A}+\mathrm{K} 9$, acetaminophen was orally administered to mice treated with $\mathrm{K} 9$; Con, control group. Data are shown as the mean \pm S.D. $(n=5)$. 
However, $\beta$-glucuronidase activities were reduced by treatment with $\mathrm{K} 8$ or K9 (Fig. 5). Subsequently, the effects of probiotics on the intestinal $\beta$-glucuronidase, sulfatase, and arylsulfate sulfotransferase activities were investigated. Figure 6 showed that the treatment with K8 or K9 did not affect the intestinal metabolic enzyme activities. This finding suggests that the alteration of acetaminophen metabolism observed in the K8-treated mice may be due to the modulation of gut microbial enzyme activities rather than the modulation of the intestinal enzyme activities.

Effects of Probiotics on the Population of Gut Microbiota. Next, by qPCR, we analyzed the composition of fecal microbiota in control and probiotic-treated mice (Fig. 7). At the phylum level, probiotic treatment with $\mathrm{K} 8$ significantly increased the number of Cyanobacteria, whereas K9 treatment increased the number of Deferribacteres.

To investigate whether orally administered probiotics adhered into the GI tract, we orally administered probiotics to mice and analyzed the amount of K8 or K9 in the epithelia of the stomach and upper small intestine using PCR. Treatment with K8 increased the number of $L$. reuteri adhered in the upper small intestine, whereas the number of $L$. rhamnosus was not affected by treatment with $\mathrm{K} 8$ or K9.

\section{Discussion}

Acetaminophen is catalyzed to hydrophilic metabolites in the intestinal mucosae and liver by phase II enzymes including sulfotransferase and UDP-glucuronyl transferase (Goon and Klaassen, 1990). These enzymes, respectively, require 3 '-phosphoadenosine- 5 ' -phosphosulfate and UDP-D-glucuronic acid as donor substrates. These reactions are limited, apparently because of the depletion of donor substrates such as glutathione (Chen et al., 2013). Nonconjugated acetaminophen $(<10 \%)$ is transformed into ABQ by the cytochrome P450 enzymes (CYP2E1, CYP1A2, and CYP3A4). ABQ, a reactive electrophile, is detoxified by conjugation with glutathione. However, when glutathione levels are depleted, ABQ binds to macromolecules such as protein and DNA in hepatocytes, resulting in liver necrosis (Hjelle and Klaassen, 1984; Hjelle et al., 1985). Moreover, the excretion of acetaminophen conjugates such as acetaminophen- $O$-sulfate in the urine is affected by antibiotics treatment (Kim and Kobashi, 1986; Gauhar et al., 2014), and its excretion into the urine is competitive with cresol- $O$-sulfate (Clayton et al., 2009). Cresol and acetaminophen are sulfated by liver arylsulfotransferase or bacterial arylsulfate sulfotransferase and are glucuronated

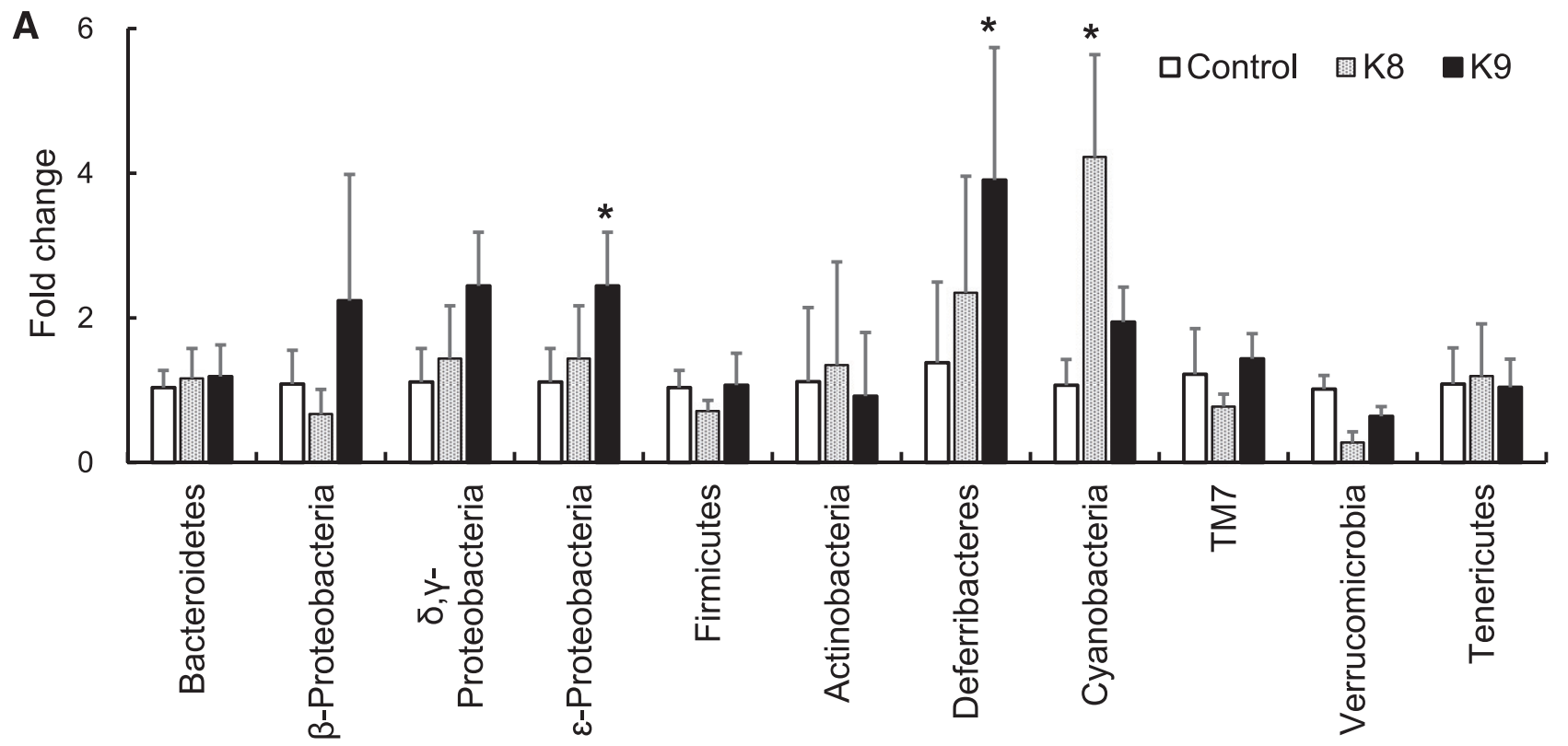

B

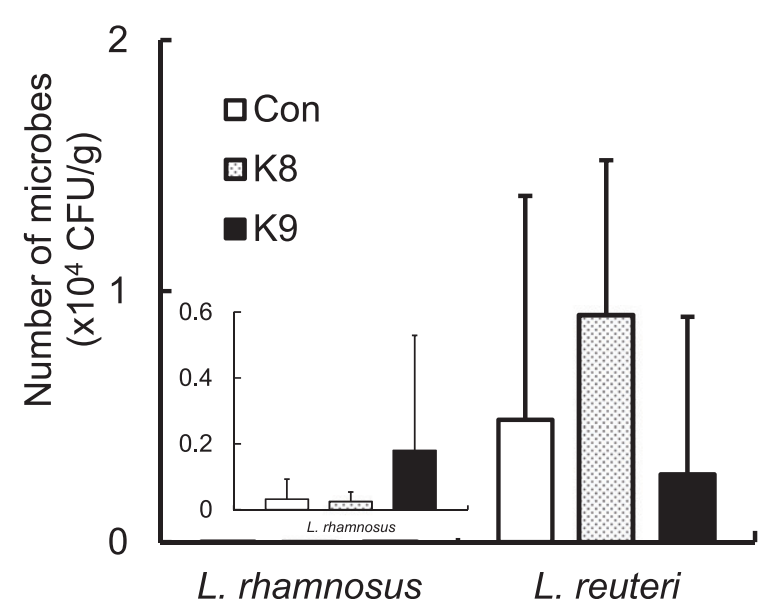

C

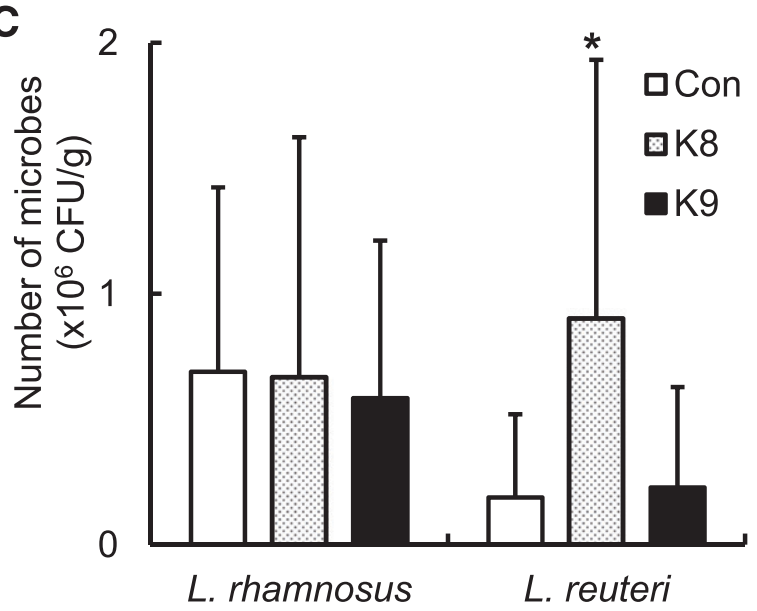

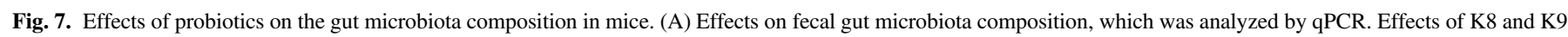

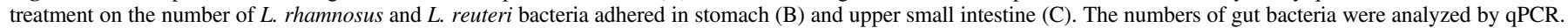
Data are shown as the mean \pm S.D. $(n=6)$. $* P<0.05$ vs. control group. 
by liver UDP-glucuronidase (Goon and Klaassen, 1990; Kim et al., 1992). Orally administered acetaminophen is mainly conjugated by sulfation, and acetaminophen-sulfoconjugates are mainly excreted into the intestine via the bile duct and urine (Kim and Kobashi, 1986; Kim et al., 1992). The conjugated acetaminophens, including acetaminophen-sulfoconjugates, are retoxified by $\beta$-glucuronidase and sulfatase of the liver and bacteria (Lee et al., 2003).

In the intestine, acetaminophen conjugates excreted via the bile duct are hydrolyzed to acetaminophen by bacterial $\beta$-glucuronidase and sulfatase (Kim and Kobashi, 1986; (Lee et al., 2012b). However, for acetaminophen conjugates, sulfoconjugates may be a donor substrate for bacterial arylsulfate sulfotransferase, which catalyzes the transfer of sulfoconjugates to phenolic compounds and produces free acetaminophen (Kim et al., 1992; Kwon et al., 1999). Meanwhile, the free acetaminophen can be sulfated by bacterial arylsulfate sulfotransferase (Kim and Kobashi, 1986). In the present study, we found that the fecal arylsulfate sulfotransferase activity in K8-treated mice is greater than that in control mice. This finding reveals the possibility that acetaminophen might be sulfoconjugated by bacterial arylsultransferase prior to being absorbed, which might limit the absorption of acetaminophen. In this context, the sulfation of free acetaminophen in the GI tract may be dependent on the concentration of phenyl sulfate esters such as cresol- $O$ sulfate and bacterial arylsulfate sulfotransferase (Kim and Kobashi, 1986; Kim et al., 1992). Accordingly, the absorption of deconjugated acetaminophen from the intestine into the blood may be dependent on the concentration of phenolic compounds and arylsulfate sulfotransferase-producing bacteria. This can be supported by reports that treatment with antibiotics decreased the number of arylsulfate sulfotransferase-producing bacteria in the GI tract and inhibited the excretion of acetaminophen into the urine (Kim and Kobashi, 1986; Gauhar et al., 2014). These results suggest that the factors that change gut microbiota composition could affect the pharmacokinetics of acetaminophen. Our results on pharmacokinetic parameters also showed that $\mathrm{K} 8$ treatment increased the oral clearance of acetaminophen and decreased the systemic exposure of acetaminophen.

Interestingly, the acetaminophen metabolite profile data showed that the portion of the urinary acetaminophen-cysteine-conjugated metabolite was increased in K8-treated mice. This result suggests the possibility that $\mathrm{K} 8$ treatment might promote the clearance of acetaminophen through glutathione conjugation to decrease the plasma levels of acetaminophen. Lutgendorff et al. (2008) reported that probiotics enhance pancreatic glutathione biosynthesis and increase plasma gluthathione levels. This report may partly explain the mechanisms for the increase of the cysteine-conjugated metabolite in K8-treated mice. Further study should be followed to clarify its underlying mechanisms.

In the present study, we found that orally administered probiotics K8 and $\mathrm{K} 9$ attached in the GI tract. Of these, $\mathrm{K} 8$ resided in the upper small intestine, not the stomach, in more significant amounts than K9. These probiotics also caused the composition of gut microbiota to fluctuate; the probiotics increased Firmicutes and Bacteroides phyla in the colon but inhibited the Proteobacteria phylum. These probiotics also changed the activities of their drug-metabolizing enzymes such as $\beta$-Dglucuronidase and arylsulfate sulfotransferase. Of these probiotics, K8 increased the degradation of acetaminophen by itself and significantly increased gut bacterial acetaminophen-degrading and arylsulfate sulfotransferase activities without changing the intestinal metabolic activities. These results suggest that probiotics could affect the absorption of acetaminophen as well as the sulfoconjugate level of acetaminophen in the intestine by modulating the gut microbial metabolic activities. Oral administration of acetaminophen in mice treated with $\mathrm{K} 8$ significantly reduced the AUC of the acetaminophen compared with that in mice treated in the absence of $\mathrm{K} 8$. These results suggest that $\mathrm{K} 8$ may inhibit the absorption into the blood of acetaminophen from the GI tract including the stomach 1) by promoting the metabolism of acetaminophen directly or via modulation gut microbial enzyme activities or 2) by increasing its sulfoconjugation in the GI tract. In addition, the data from Table 3 suggest a possibility that the decrease of the bowel transit time due to stimulation of the peristaltic movements by K8 might disturb the absorption of acetaminophen. If sustained-release acetaminophen is used for treatment, the sulfoconjugation of orally administered acetaminophen may increase in the GI tract, which may lead to a decrease in the absorption of the acetaminophen.

\section{Authorship Contributions}

Participated in research design: Yoo and D.-H. Kim.

Conducted experiments: J.-K. Kim, Choi, Jeong, and Lim.

Contributed new reagents or analytic tools: I.S. Kim.

Performed data analysis: J.-K. Kim, Choi, and Lim.

Wrote or contributed to the writing of the manuscript: J.-K. Kim, Choi, Yoo, and D.-H. Kim.

\section{References}

Al-Hilal TA, Alam F, and Byun Y (2013) Oral drug delivery systems using chemical conjugates or physical complexes. Adv Drug Deliv Rev 65:845-864.

An JH, Lee HJ, and Jung BH (2012) Quantitative analysis of acetaminophen and its six metabolites in rat plasma using liquid chromatography/tandem mass spectrometry. Biomed Chromatogr 26: 1596-1604.

Boles JW and Klaassen CD (2000) Effects of molybdate and pentachlorophenol on the sulfation of acetaminophen. Toxicology 146:23-35.

Bron PA, Kleerebezem M, Brummer RJ, Cani PD, Mercenier A, MacDonald TT, Garcia-Ródenas CL, and Wells JM (2017) Can probiotics modulate human disease by impacting intestinal barrier function? Br J Nutr 117:93-107.

Chen Y, Dong H, Thompson DC, Shertzer HG, Nebert DW, and Vasiliou V (2013) Glutathione defense mechanism in liver injury: insights from animal models. Food Chem Toxicol 60:38-44. Clayton TA, Baker D, Lindon JC, Everett JR, and Nicholson JK (2009) Pharmacometabonomic identification of a significant host-microbiome metabolic interaction affecting human drug metabolism. Proc Natl Acad Sci USA 106:14728-14733.

Davis SS (2005) Formulation strategies for absorption windows. Drug Discov Today 10:249-257. Gauhar S, Ali SA, Naqvi SB, and Shoaib MH (2014) Report: pharmacokinetic and drug interaction studies of pefloxacin with paracetamol (NNAID) in healthy volunteers in Pakistan. Pak J Pharm Sci 27:389-395.

Goldin BR and Gorbach SL (2008) Clinical indications for probiotics: an overview. Clin Infect Dis 46 (Suppl 2):S96-S100, discussion S144-S151.

Goon D and Klaassen CD (1990) Dose-dependent intestinal glucuronidation and sulfation of acetaminophen in the rat in situ. J Pharmacol Exp Ther 252:201-207.

Hjelle JJ, Hazelton GA, and Klaassen CD (1985) Acetaminophen decreases adenosine 3'-phosphate 5'-phosphosulfate and uridine diphosphoglucuronic acid in rat liver. Drug Metab Dispos 13:35-41.

Hjelle JJ and Klaassen CD (1984) Glucuronidation and biliary excretion of acetaminophen in rats. $J$ Pharmacol Exp Ther 228:407-413.

Joh EH and Kim DH (2010) A sensitive liquid chromatography-electrospray tandem mass spectrometric method for lancemaside A and its metabolites in plasma and a pharmacokinetic study in mice. J Chromatogr B Analyt Technol Biomed Life Sci 878:1875-1880.

Kato R, Yuasa H, Inoue K, Iwao T, Tanaka K, Ooi K, and Hayashi Y (2007) Effect of Lactobacillus casei on the absorption of nifedipine from rat small intestine. Drug Metab Pharmacokinet 22:96-102.

Kim DH (2015) Gut Microbiota-mediated drug-antibiotic interactions. Drug Metab Dispos 43: 1581-1589.

Kim DH, Hyun SH, Shim SB, and Kobashi K (1992) The role of intestinal bacteria in the transformation of sodium picosulfate. Jpn J Pharmacol 59:1-5.

Kim DH and Kobashi K (1986) The role of intestinal flora in metabolism of phenolic sulfate esters. Biochem Pharmacol 35:3507-3510.

Klaassen CD and Cui JY (2015) Review: mechanisms of how the intestinal microbiota alters the effects of drugs and bile acids. Drug Metab Dispos 43:1505-1521.

Kwon AR, Oh TG, Kim DH, and Choi EC (1999) Molecular cloning of the arylsulfate sulfotransferase gene and characterization of its product from Enterobacter amnigenus AR-37. Protein Expr Purif 17:366-372.

Lee HJ, Zhang H, Orlovich DA, and Fawcett JP (2012a) The influence of probiotic treatment on sulfasalazine metabolism in rat. Xenobiotica 42:791-797.

Lee HW, Choo MK, Bae EA, and Kim DH (2003) Beta-glucuronidase inhibitor tectorigenin isolated from the flower of Pueraria thunbergiana protects carbon tetrachloride-induced liver injury. Liver Int 23:221-226.

Lee SH, An JH, Lee HJ, and Jung BH (2012b) Evaluation of pharmacokinetic differences of acetaminophen in pseudo germ-free rats. Biopharm Drug Dispos 33:292-303.

Lennernäs H and Abrahamsson B (2005) The use of biopharmaceutic classification of drugs in drug discovery and development: current status and future extension. J Pharm Pharmacol 57: 273-285.

Lutgendorff F, Trulsson LM, van Minnen LP, Rijkers GT, Timmerman HM, Franzén LE, Gooszen HG, Akkermans LM, Söderholm JD, and Sandström PA (2008) Probiotics enhance pancreatic glutathione biosynthesis and reduce oxidative stress in experimental acute pancreatitis. Am J Physiol Gastrointest Liver Physiol 295:G1111-G1121.

Mitchell JR, Jollow DJ, Potter WZ, Gillette JR, and Brodie BB (1973) Acetaminophen-induced hepatic necrosis. IV. Protective role of glutathione. J Pharmacol Exp Ther 187:211-217. 
Saad R, Rizkallah MR, and Aziz RK (2012) Gut pharmacomicrobiomics: the tip of an iceberg of complex interactions between drugs and gut-associated microbes. Gut Pathog 4:16-28.

Sousa T, Paterson R, Moore V, Carlsson A, Abrahamsson B, and Basit AW (2008) The gastrointestinal microbiota as a site for the biotransformation of drugs. Int J Pharm 363:1-25.

Stojančević M, Bojić G, Salami HA, and Mikov M (2014) The influence of intestinal tract and probiotics on the fate of orally administered drugs. Curr Issues Mol Biol 16:55-68.

Tralau T, Sowada J, and Luch A (2015) Insights on the human microbiome and its xenobiotic metabolism: what is known about its effects on human physiology? Expert Opin Drug Metab Toxicol 11:411-425.

Wasilewski A, Zielińska M, Storr M, and Fichna J (2015) Beneficial effects of probiotics, prebiotics, synbiotics, and psychobiotics in inflammatory bowel disease. Inflamm Bowel Dis 21 $1674-1682$.

Watari N, Iwai M, and Kaneniwa N (1983) Pharmacokinetic study of the fate of acetaminophen and its conjugates in rats. J Pharmacokinet Biopharm 11:245-272.
Yang YW, Chen MK, Yang BY, Huang XJ, Zhang XR, He LQ, Zhang J, and Hua ZC (2015) Use of 16S rRNA gene-targeted group-specific primers for real-time PCR analysis of predominant bacteria in mouse feces. Appl Environ Microbiol 81:6749-6756.

Address correspondence to: Dr. Hye Hyun Yoo, Institute of Pharmaceutical Science and Technology and College of Pharmacy, Hanyang University, Ansan, Gyeonggi-do 15588, Korea. E-mail: yoohh@hanyang.ac.kr or Dr. Dong-Hyun Kim, Department of Life and Nanopharmaceutical Sciences, College of pharmacy, Kyung Hee University, 26, Kyungheedae-ro, Dongdaemun-gu, Seoul 02447, Korea. E-mail: dhkim@khu.ac.kr 